\title{
Increased Susceptibility to Apoptosis and Growth Arrest of Human Breast Cancer Cells Treated by a Snake Venom-Loaded Silica Nanoparticles
}

\author{
Gamal Badra Douaa Sayed ${ }^{\mathrm{b}}$ Doaa Maximous ${ }^{\mathrm{c}}$ Amany O. Mohamed ${ }^{\mathrm{d}}$ \\ Mustafa Gule
}

aLaboratory of Immunology \& Molecular Biology, Zoology Department, Faculty of Science, Assiut University, ${ }^{b} C$ linical Pathology Department, South Egypt Cancer Institute, Assiut University, 'Surgical Oncology Department, South Egypt Cancer Institute, Assiut University, dDepartment of Biochemistry,

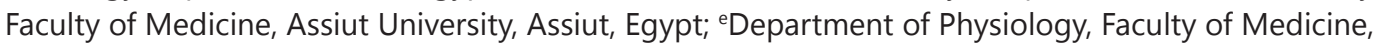
Ataturk University, Erzurum, Turkey

\section{Key Words}

Apoptosis • Breast cancer $•$ Nanoparticles $•$ Proliferation $\bullet$ Snake venom

\begin{abstract}
Background: The development of effective treatments against metastatic cancers, including breast cancer, is among the most important challenges in current experimental and clinical cancer research. We recently demonstrated that Walterinnesia aegyptia venom (WEV), either alone or in combination with silica nanoparticles (WEV+NP), resulted in the growth arrest and apoptosis of different cancer cell lines. Aims: In the present study, we evaluated the impact of WEV alone and WEV+NP on human breast cancer cells isolated from cancer biopsies. Methods: The potential effects of WEV alone and WEV+NP on the proliferation, induction of apoptosis and generation of free radicals in breast cancer cells isolated from 80 patients clinically diagnosed with breast cancer were evaluated by flow cytometry and ELISA. Results: WEV alone and WEV+NP inhibited the proliferation, altered the cell cycle and enhanced the induction of apoptosis of the breast cancer cells by increasing the activities of caspase-3, caspase- 8 and caspase- 9 . In addition, the combination of WEV and NP robustly sensitized the breast cancer cells to growth arrest and apoptosis by increasing the generation of free radicals, including reactive oxygen species (ROS), hydroperoxide and nitric oxide. The combination of WEV with NP significantly enhanced the anti-tumor effect of WEV in breast cancer cells. Conclusion: Our data indicate the therapeutic potential of the nanoparticle-sustained delivery of snake venom for the treatment of breast cancer.
\end{abstract}




\section{Introduction}

Despite major advances in the elucidation of the mechanisms of breast cancer progression and the development of novel therapeutic agents, breast cancer remains the second leading cause of mortality among women worldwide [1]. This mortality is almost invariably due to metastasis $[1,2]$. Metastatic disease remains the most critical factor limiting patient survival, and the development of effective treatments against metastatic cancers, including breast cancer, is among the most important challenges in current experimental and clinical cancer research [3-5].

Natural toxins are recognized as sources for drugs against several human ailments, including cancers. In particular, a non-toxic dose of snake venom has been shown to both reduce the size of solid tumors and block the process of angiogenesis in ovarian cancer [6]. New anticancer agents must be identified to increase the number of available options and identify less toxic and more effective drugs. Snake venom is a complex mixture of many substances, including toxins, enzymes, growth factors, activators and inhibitors, with a wide spectrum of biological activities. Our recent studies have demonstrated the antitumor potential of snake venom from Walterinnesia aegyptia (WEV) on the human breast carcinoma cell line MDA-MB-231 and have shown its effect on normal murine peripheral blood mononuclear cells (PBMCs) [7]. In addition, other data have indicated that the snake venom toxin from Vipera lebetina turanica inhibits hormone-refractory human prostate cancer cell growth at nanogram concentrations; this effect is related to the NF- $\kappa \mathrm{B}$ signalmediated induction of apoptosis [8].

One mechanism by which chemotherapeutic agents kill tumor cells is by inducing apoptotic death pathways. The ability of cancer cells to escape from apoptosis and continue to proliferate is one of the fundamental hallmarks of cancer and is a major target of cancer treatment; therefore, the underlying mechanisms of apoptosis and cancer progression continue to be a focus of intense research. In a cell, apoptosis can be triggered through either the extrinsic pathway or the intrinsic pathway. In the extrinsic pathway, signal molecules, known as ligands, bind to transmembrane death receptors on the target cell to induce apoptosis through the activation of cellular caspases, while the intrinsic pathway is triggered by cellular stress and is mediated through a mitochondrial-dependent pathway. Caspasedependent apoptosis includes the activation of caspase- 3 , caspase- 8 and caspase-9, whereas the mitochondrial pathway involves the efflux of cytochrome $C$ from the mitochondria to the cytosol to form apoptosomes with Apaf-1 and caspase-9, which lead to the activation of caspase-3 and subsequent apoptosis induction $[9,10]$. In fact, both pathways are intricately related. Tumor cells contain fewer scavengers of free radicals than normal cells, and free radicals have been shown to participate in the mechanism of anticancer therapeutic agents; the production of large amounts of free radicals in tumor tissues may therefore have potential as a future anticancer therapy [11]. Consequently, modulation of the levels of reactive oxygen species (ROS) and other free radicals that induce oxidative stress has been proposed as a therapeutic approach to cancer [12]. Nitric oxide (NO) plays important physiological roles in vascular function and the inflammatory response. However, NO over-production induces DNA damage, mitochondrial uncoupling and increased ROS [12-14].

Nanoparticles loaded with chemical therapeutics have shown great promise for the treatment of cancer. When loaded with anticancer agents, nanoparticles can successfully increase drug concentrations in cancer tissues and act at the cellular level to enhance antitumor efficacy. The nanoparticles can be endocytosed and/or phagocytosed by cells, resulting in the internalization of the encapsulated drug [9]. Therefore, in the present study, we investigated the effects of WEV alone and in combination with silica nanoparticles $(\mathrm{WEV}+\mathrm{NP})$ on the proliferation and apoptosis of human breast cancer cells through monitoring the caspase activity and free radical levels. 
Badr et al.: Snake Venom-Loaded Silica Nanoparticles Induce Growth Arrest in Human Breast Cancer Cells

\section{Materials and Methods}

\section{Preparation of Walterinnesia aegyptia venom}

Walterinnesia aegyptia snakes were collected from the central region of Saudi Arabia. No specific permits were required for the described field studies, and no specific permission was required for these locations/activities because the location was not privately owned or protected in any way and the field studies did not involve endangered or protected species. The snakes were kept in a serpentarium in the Zoology Department of the College of Science at King Saud University. The snakes were warmed daily for nine hours using a 100-watt lamp and were provided water ad libitum. The snakes were fed purpose-bred mice every 10 to 14 days. After the venom was milked from a single specimen of adult snake, the venom was lyophilized and reconstituted in $1 \mathrm{X}$ phosphate-buffered saline (PBS) prior to use.

\section{Combination of snake venom with silica nanoparticles}

As previously described $[7,15]$, a double mesoporous core-shell silica nanosphere was formed around a silica core by using an anionic surfactant to transform the solid silica core into a mesoporous core. To synthesize the solid silica core, $0.875 \mathrm{ml}$ aqueous ammonia was added to a solution that contained $18 \mathrm{ml}$ ethanol and $2.6 \mathrm{ml}$ deionized water; then, $1.5 \mathrm{ml}$ tetraethyl orthosilicate (TEOS) was added while the solution was vigorously stirred. The resulting mixture was heated to $30^{\circ} \mathrm{C}$ for $60 \mathrm{~min}$, and the silica precipitate was then collected by centrifugation and washed three times with water. Second, to synthesize the mesoporous core-shell nanosphere, silica $\left(\mathrm{SiO}_{2}\right)$ particles were dispersed using an anionic surfactant in $15 \mathrm{ml} \mathrm{H}_{2} \mathrm{O}$ and ultrasonicated for $10 \mathrm{~min}$. To suppress the agglomeration of the silica cores, $1 \mathrm{~g} / \mathrm{l}$ polyvinylpyrrolidone was added followed by constant stirring for $60 \mathrm{~min}$. Next, $0.1 \mathrm{ml} \mathrm{3-aminopropyltrimethoxysilane} \mathrm{(APMS),}$ $0.2933 \mathrm{~g} \mathrm{(1} \mathrm{mmol)} \mathrm{N-lauroylsarcosine} \mathrm{sodium} \mathrm{(Sar-Na)} \mathrm{and} 1.5 \mathrm{ml}$ TEOS were added to the reaction mixture, which was stirred at $50^{\circ} \mathrm{C}$ for $2 \mathrm{~h}$. The final solid was recovered by centrifugation, washed with deionized water and dried in an oven at $60^{\circ} \mathrm{C}$ for $12 \mathrm{~h}$. Template removal was performed by heat treatment in an air stream at $550^{\circ} \mathrm{C}$ for 6 hours. After synthesizing the nanoparticles, $25 \mathrm{mg}$ mesoporous silica nanoparticles was added to a solution of $50 \mathrm{mg} / \mathrm{ml}$ venom in $0.5 \mathrm{ml}$ water. The suspension was stirred for 2 hours, and the evaporation of water was prevented. The mesoporous silica nanoparticles loaded with venom were recovered by high-speed centrifugation and then dried in a vacuum oven at $60^{\circ} \mathrm{C}$. Transmission electron microscopy (TEM) was performed with a JEOL JSM-2100F electron microscope (Japan) operated at 200 $\mathrm{kV}$. Nitrogen sorption isotherms were measured at $77 \mathrm{~K}$ with a Quantachrome NOVA 4200 analyzer (USA). Prior to taking measurements, the samples were degassed in a vacuum at $200^{\circ} \mathrm{C}$ for at least 18 hours. The Brunauer-Emmett-Teller (BET) method was utilized to calculate the specific surface areas (SBET) using adsorption data in a relative pressure range from 0.05 to 0.35. Using the Barrett-Joyner-Halenda (BJH) model, the pore volumes and pore size distributions were derived from the adsorption branches of the isotherms, and the total pore volumes $(V t)$ were estimated from the adsorbed amount at a relative pressure $(P / P 0)$ of 0.992 .

\section{Human breast cancer samples}

Breast cancer tissue samples were obtained from 80 females with histologically proven breast cancer. The clinico-histopathological data of the patients are summarized in Table 1. All patients had been surgically treated at the South Egypt Cancer Institute of Assiut University in Egypt. Non-tumorigenic normal breast tissue samples were obtained from Assiut University Hospital. The tumor tissue specimens were taken at the time of surgery after informed written consent in accordance with South Egypt Cancer Institute ethical committee guidelines. This study was approved by the Ethical committee of South Egypt Cancer Institute, Assiut University, Egypt. This ethical committee is approved by U.S. Department of Health and Human Services (HHS) Institutional Review Board (IRB). IORG number: IORG0006563. OMB number: 09900279. The tumor and normal tissues were immediately disaggregated mechanically by passage through a 16-gauge stainless steel mesh, and the cells were either lysed in lysis buffer for the caspase activity test and the measurement of free radical levels or directly transferred to and maintained in a culture medium consisting of MEM supplemented with 10\% heat-inactivated fetal bovine serum (FBS, EuroClone, Life Science Division, Milan, Italy). The anti-proliferative effects of WEV, NP and WEV+NP were determined on the non-tumorigenic and breast cancer cells isolated from human samples using the 3-(4,5-dimethylthiazol2-yl)-2, 5-diphenyltetrazolium bromide (MTT) uptake method. The cells were plated at $1 \times 10^{6} \mathrm{cells} / \mathrm{ml}$ in 2 
Badr et al.: Snake Venom-Loaded Silica Nanoparticles Induce Growth Arrest in Human Breast Cancer Cells

ml culture medium in six-well Costar plates (Corning, Corning, NY). The cells were treated with different concentrations of WEV or WEV+NP for $1,2,6,12,24$ or 48 h. Cytotoxicity was expressed as the relative percentage of the OD values measured in the control untreated (0), NP-, WEV- and WEV+NP-treated cells. Morphological changes following exposure to NP, WEV and WEV+NP were observed using a phase contrast inverted microscope (Olympus, Japan).
Table 1. Clinico-histopathological data for patients diagnosed with breast cancer

\begin{tabular}{llc}
\hline Clinico-histopathological parameters & Patients (No.) \\
\hline Histopathology & Infiltrating ductal carcinoma & 61 \\
& Infiltrating lobular carcinoma & 14 \\
& Other & 5 \\
Grade & GII & 53 \\
& GIII & 27 \\
Lymph node involvement & N0 & \\
& N1 & 23 \\
& N2 & 49 \\
& N3 & 5 \\
Tumor burden & T2 & 3 \\
& T3 & 55 \\
& T4 & 20 \\
\hline
\end{tabular}

CFSE proliferation assay and flow cytometry analysis

Flow cytometry was performed with the FACSCalibur system (BD, San Jose, CA). All fluorocytometric data were subsequently analyzed and displayed with CELL QUEST software (BD, San Jose, CA). Each analysis included measurements for a minimum of 20,000 cells. The breast cancer cells were washed twice in PBS and stained with $0.63 \mu \mathrm{M}$ carboxyfluorescein diacetate succinimidyl ester (CFSE) (Molecular Probes, Eugene, OR) for $8 \mathrm{~min}$ at room temperature. The residual CFSE was removed by washing 3 times in PBS. The CFSElabeled cells were then seeded into 6-well plates, treated with NP, WEV or WEV+NP or left untreated (0) and grown for 4 days in RPMI cell culture medium. The cells were then stained with monoclonal antibodies. The CFSE fluorescence intensity was measured by flow cytometry. Isotype control antibodies were used to separate the positive and negative cells.

Flow cytometry analysis of apoptosis and cell cycle analysis

After treatment with NP, WEV or WEV+NP, the breast cancer cells were fixed and permeabilized with $70 \%$ ice-cold ethanol for at least $1 \mathrm{~h}$ and then washed twice with PBS. The DNA was stained by incubating the cells at $37^{\circ} \mathrm{C}$ for $1 \mathrm{~h}$ in $40 \mu \mathrm{g} / \mathrm{ml}$ propidium iodide and $100 \mu \mathrm{g} / \mathrm{ml}$ DNase-free RNase in PBS. The fluorescence area (FL2-A) is the main parameter in the cell cycle analysis; therefore, a histogram plot of FL2-A was used as a cell cycle graph. The cell cycle distributions were analyzed with Modfit LT 3.0 software (BD, San Jose, CA). Dead cells were identified using the Trypan blue dye exclusion test. The breast cancer cells were reanalyzed for the expression of annexin $\mathrm{V}$ and both annexin $\mathrm{V}$ and PI to identify early and late apoptosis, respectively.

\section{Measurements of caspase activity}

Caspase-3, caspase-8 and caspase- 9 activities were evaluated using a fluorometric protease assay kit (MBL, Aichi, Japan) according to the manufacturer's instructions.

Measurements of ROS, hydroperoxide and nitric oxide levels

The levels of ROS were determined using 2,7-dichlorodihydrofluorescein diacetate (H2DCF-DA) (Beyotime Institute of Biotechnology, Haimen, China). Tumor cells $\left(1 \times 10^{6}\right)$ were directly treated with 10 $\mu \mathrm{M}$ H2DCF-DA dissolved in $1 \mathrm{ml} \mathrm{PBS}$ at $37^{\circ} \mathrm{C}$ for $20 \mathrm{~min}$. The fluorescence intensity was monitored with an excitation wavelength of $488 \mathrm{~nm}$ and an emission wavelength of $530 \mathrm{~nm}$. The levels of hydroperoxide were measured using the free radical analytical system (FRAS 2, Iram, Parma, Italy). This test is a colorimetric assay that takes advantage of the ability of hydroperoxide to generate free radicals after reaction with specific transition metals. The concentrations of nitrite and nitrate were measured with a Griess assay reagent $\left(\mathrm{NO}_{2} / \mathrm{NO}_{3}\right.$ detection kit; Dojindo, Kumamoto, Japan) according to the manufacturer's instructions. In brief, the supernatant of the cell culture medium without phenol or serum was collected and then reacted with the Griess reagent. The azo coupling between the diazonium species, which are produced by the reaction of sulfanilamide with NO2, and 1-naphthylethylenediamine was measured at $540 \mathrm{~nm}$ with an MRX microplate reader (Dynex Technologies, Inc., Chantilly, VA). 
Badr et al.: Snake Venom-Loaded Silica Nanoparticles Induce Growth Arrest in Human Breast Cancer Cells

Fig. 1. WEV and WEV+NP inhibit the growth of breast cancer cells. Breast cancer cells isolated from human subjects were treated with NP (open circle), WEV (gray triangles) or WEV+NP (closed squares) at concentrations of $0,1,5,10,20,50,100$ and 1000 $\mathrm{ng} / \mathrm{ml}(\mathrm{A})$ and different incubation times of $0,1,2,6$, 12, 24 and $48 \mathrm{hr}$ (B); cell viability was assessed using an MTT assay. The combined data from different experiments $(n=5)$ are shown, and the results are expressed as the mean percentage of viable cells \pm SEM. Similarly, non-tumorigenic normal breast cells were treated with the same concentrations of NP, WEV and WEV+NP (C). The pooled data from different experiments $(n=5)$ are shown, and the results are expressed as the mean percentage of viable cells \pm SEM.

\section{Statistical analysis}

The data were first tested for normality using the Anderson-Darling test and then evaluated for variance homogeneity prior to further statistical analysis. The data were normally distributed and were expressed as the mean \pm standard error of the mean (SEM). Significant differences among groups were analyzed by one- or two-way ANOVA followed by the Bonferroni multiple comparison test using PRISM statistical analysis software (GraphPad Software). The data were then reanalyzed by oneor two-way ANOVA followed by Tukey's range test using SPSS software, version 17. Differences were considered significant at $\mathrm{P}<0.05$. ${ }^{*} \mathrm{P}<0.05$, WEVtreated vs. control; $\# \mathrm{P}<0.05$, WEV+NP-treated vs. control; $+\mathrm{P}<0.05$, WEV+NP-treated vs. WEV-treated.

\section{Results}
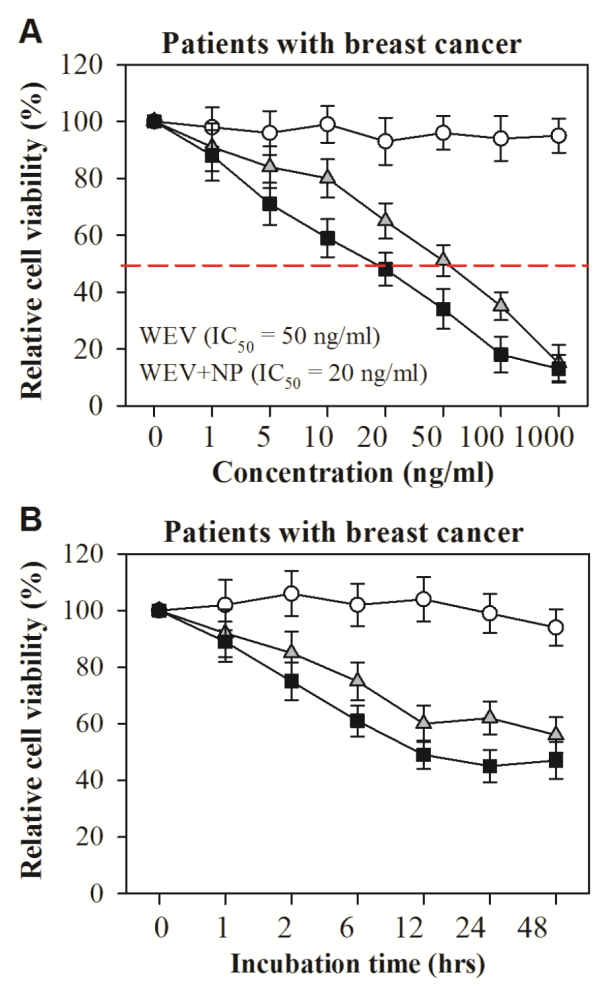

C

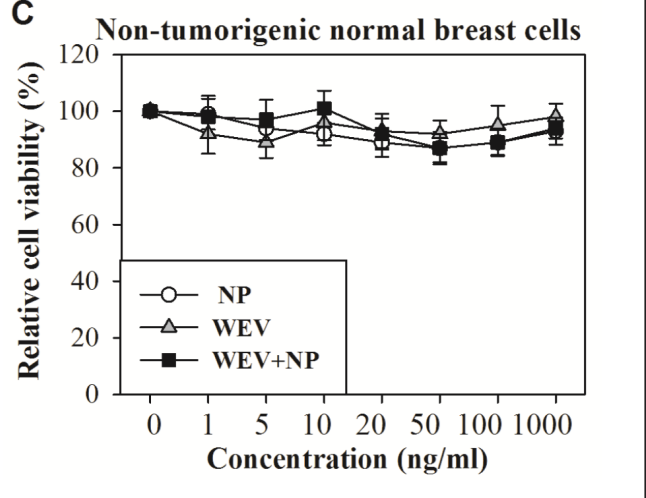

WEV and WEV+NP inhibit the growth of breast cancer cells

Using a silica nanoparticles delivery system, we first investigated the ability of WEV and $\mathrm{WEV}+\mathrm{NP}$ to induce growth arrest in breast cancer cells. The effects of WEV and WEV+NP on breast cancer cells and normal breast cells were examined at WEV concentrations of $0,1,5,10,20,50,100$ and $1000 \mathrm{ng} / \mathrm{ml}$ and incubation times of $0,1,2,6,12,24,36$ and $48 \mathrm{~h}$. The resulting cytotoxic effects of WEV and WEV+NP were measured using the MTT uptake method. The results of five independent experiments $(n=5)$ demonstrated that WEV and WEV+NP significantly inhibited the growth of breast cancer cells in a dose- and timedependent manner (Fig. 1A \& B). The $\mathrm{IC}_{50}$ values for WEV alone and WEV+NP were $50 \mathrm{ng} /$ $\mathrm{ml}$ and $20 \mathrm{ng} / \mathrm{ml}$, respectively. The effect was maximal at $12 \mathrm{~h}$ of incubation. Nevertheless, treatment with WEV or WEV+NP had no significant inhibitory effect on the viability of nontumorigenic normal breast cells (Fig. 1C). The combination of WEV with NP (WEV+NP) significantly enhanced the inhibitory effect of WEV in breast cancer cells. The maximal inhibitory effects of WEV and WEV+NP on cell viability were observed $12 \mathrm{~h}$ after treatment with $50 \mathrm{ng} / \mathrm{ml}$ of WEV alone or $20 \mathrm{ng} / \mathrm{ml}$ of WEV+NP. Nevertheless, treatment with NP alone did not affect breast cancer cell viability. 


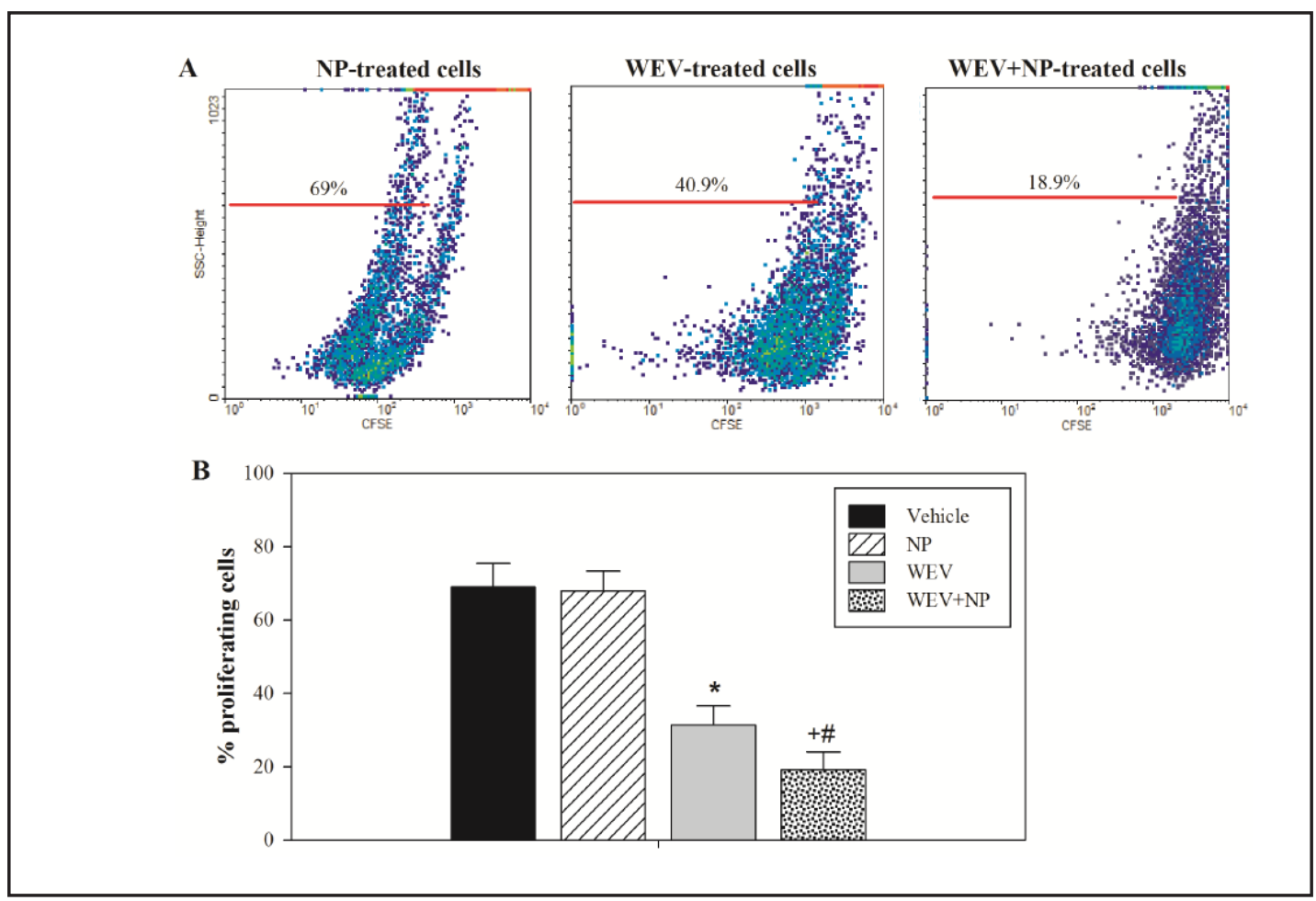

Fig. 2. WEV and WEV+NP decrease the proliferation of breast cancer cells. CFSE assays and flow cytometry were used to evaluate the ability of breast cancer cells to proliferate after treatment with vehicle, NP, WEV and WEV+NP. (A) One representative experiment showing the analysis of the CFSE staining of the breast cancer cells from one patient after gating on the viable cells. (B) The accumulated data for 80 patients are expressed as the mean percentage of proliferating cells \pm SEM for vehicle-treated cancer cells (closed black bars), NP-treated cells (hatched bars), WEV-treated cells (closed gray bars) and WEV+NP-treated cells (dotted bars). $\mathrm{P}<0.05$, WEV-treated vs. control; \#P $<0.05$, WEV+NP-treated vs. control; $+\mathrm{P}<0.05$, WEV+NPtreated vs. WEV-treated.

Treatment with WEV alone and WEV+NP inhibits the proliferation of breast cancer cells

Because the proliferation process is crucial for the maintenance and progression of cancer cells, we monitored the effects of WEV alone or in combination with NP (WEV+NP) on the proliferation of breast cancer cells using a CFSE dilution assay followed by flow cytometry. The data from one representative experiment are shown as dot plots (Fig. 2A) and reveal that the percentage of proliferating cells was markedly decreased from $69 \%$ in NP-treated cells to $40.9 \%$ and $18.9 \%$ in WEV- and WEV+NP-treated cells, respectively. The pooled data for different patients diagnosed with breast cancer $(n=80)$ demonstrated that treatment with WEV alone significantly reduced $(\mathrm{P}<0.05)$ the proliferative capacity of breast cancer cells compared with vehicle-treated cells (Fig. 2B). Moreover, although NP had no effect on proliferation, the combination of NP with WEV significantly enhanced the inhibitory effect of WEV on breast cancer cells.

Treatment with WEV and WEV+NP induces cell cycle arrest and apoptosis in breast cancer cells

The inhibition of cancer cell proliferation, the cessation of cell-cycle progression and the induction of apoptosis have all been targeted in chemotherapeutic strategies for the treatment of cancer. Therefore, we used propidium iodide (PI) single staining and PI/annexin $\mathrm{V}$ double staining followed by flow cytometry analysis to determine if WEV and WEV+NP alter the cell cycle of breast cancer cells. The data from one representative experiment are presented as a histogram; the percentage of apoptotic cells was $11 \%$ in the NP-treated cells. 


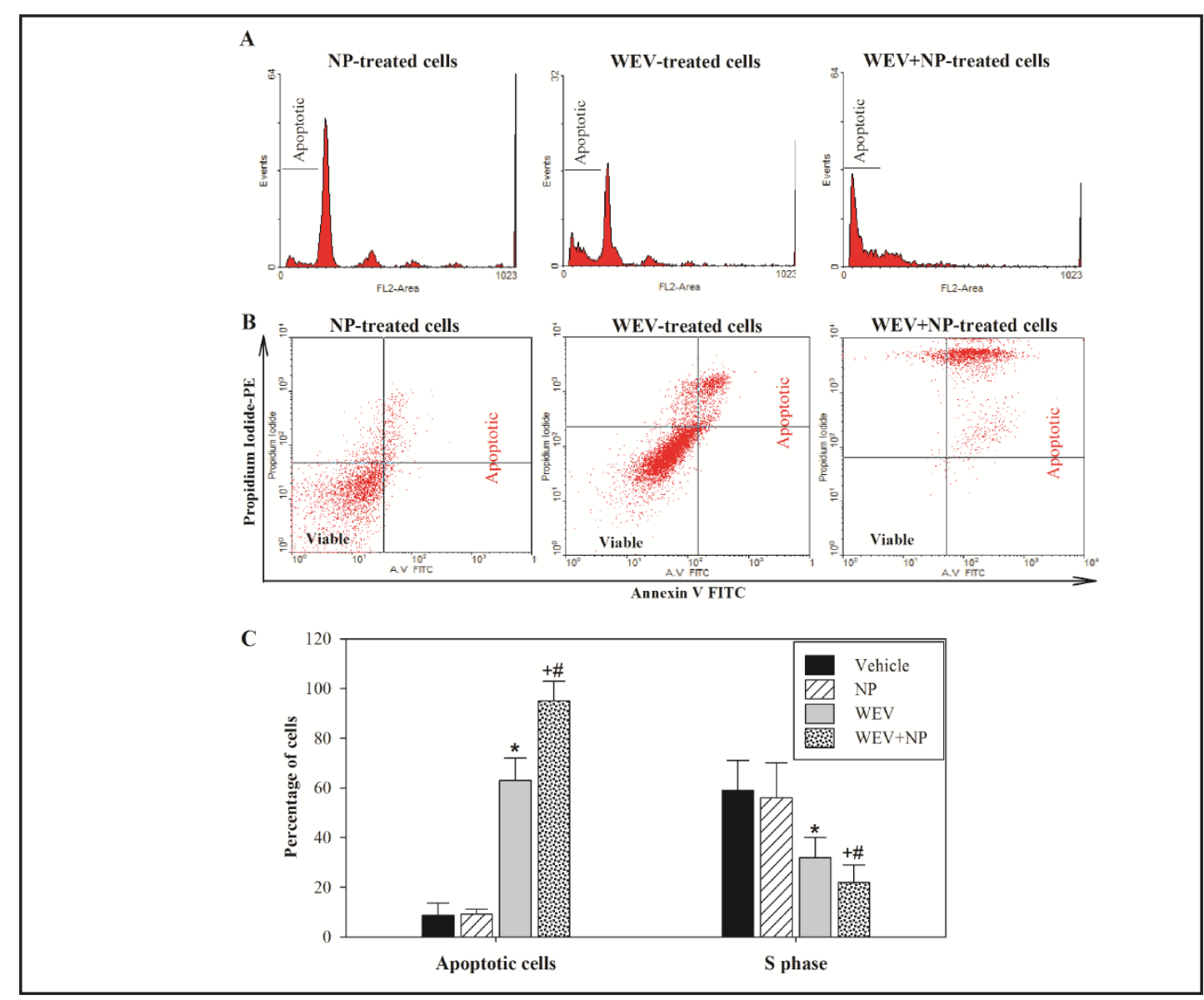

Fig. 3. The impact of WEV+NP on cell cycle and apoptosis induction in breast cancer cells. (A) The ability of WEV and WEV+NP to alter the cell cycle of breast cancer cells was evaluated by PI and flow cytometry. The PI-labeled cells were gated depending on the PI-Area and the PI-Width to calculate the G1, S, G2/M and sub-G1 (apoptotic cells) cell-cycle phases. Histograms of the PI-stained cells from one representative experiment are shown. (B) A dot plot of PI/annexin V FITC-stained cells demonstrates an increase in apoptotic breast cancer cells after treatment with NP, WEV and WEV+NP. (C) The accumulated data for 80 patients are expressed as the mean percentage \pm SEM of apoptotic and S phase cells among vehicle-treated cancer cells (closed black bars), NP-treated cells (hatched bars), WEV-treated cells (closed gray bars) and WEV+NP-treated cells (dotted bars). P $<0.05$, WEV-treated vs. control; \#P $<0.05$, WEV+NP-treated vs. control; $+\mathrm{P}<0.05$, WEV+NP-treated vs. WEV-treated.

Treatment with WEV and WEV+NP markedly increased the percentage of apoptotic cells to $35 \%$ and $85 \%$, respectively (Fig. 3A). The increased induction of apoptosis after treatment with WEV and WEV+NP was inversely correlated with a decrease in the percentage of cells in S phase to $59 \%$ and $25 \%$, respectively. The breast cancer cells were also stained with $\mathrm{PI} /$ annexin $\mathrm{V}$ to discriminate between apoptotic, necrotic and viable cells, and the data are presented as dot plots. The data from one representative experiment indicate that the percentage of apoptotic cells clearly increased in WEV- and WEV+NP-treated cells compared with NP-treated cells (Fig. 3B). The accumulated data for cells isolated from different patients $(n=80)$ demonstrated that treatment with WEV alone significantly $(P<0.05)$ potentiated apoptosis and diminished the percentage of cells in S phase in breast cancer cells compared with vehicle- and NP-treated cells (Fig. 3C). Although NP alone had no effect on apoptosis induction and the percentage of cells in S phase, the combination of NP and WEV significantly $(\mathrm{P}<0.05)$ increased apoptosis induction and decreased the percentage of cells in $\mathrm{S}$ phase. 


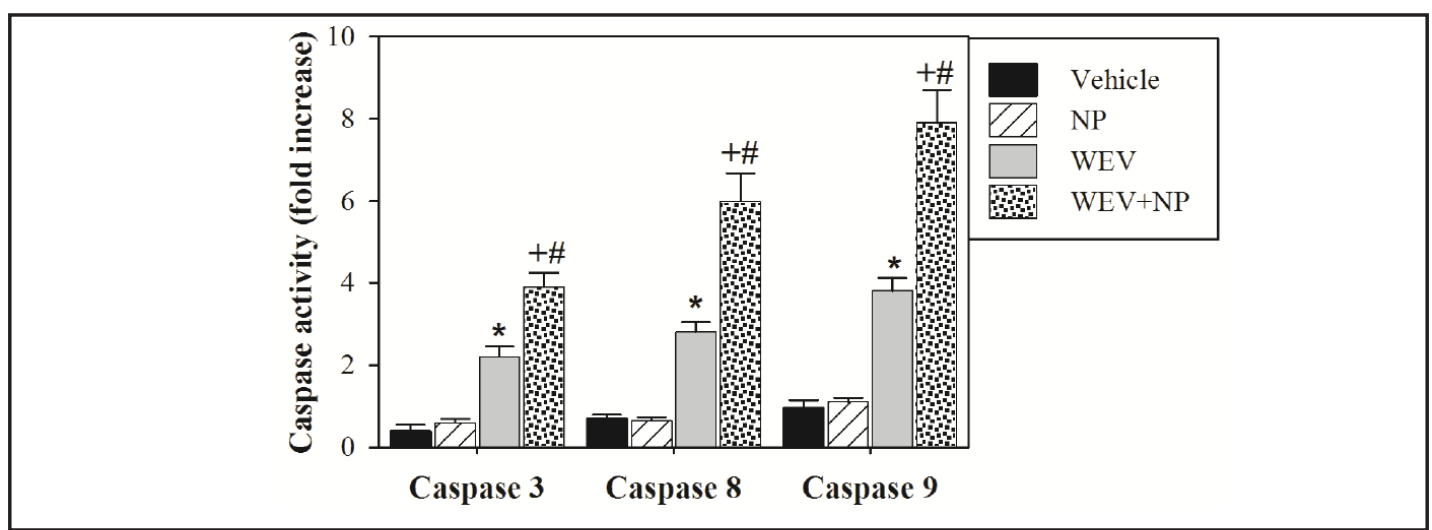

Fig. 4. The impact of WEV and WEV+NP on the levels of caspase activities in breast cancer cells. Levels of caspase-3, caspase- 8 and caspase- 9 activities were measured in the lysates of breast cancer cells after treatment with vehicle, NP, WEV or WEV+NP. The accumulated data for 80 patients are expressed as the mean fold increase \pm SEM in the caspase activity in vehicle-treated cancer cells (closed black bars), NPtreated cells (hatched bars), WEV-treated cells (closed gray bars) and WEV+NP-treated cells (dotted bars). $\mathrm{P}<0.05$, WEV-treated vs. control; \#P $<0.05$, WEV+NP-treated vs. control; $+\mathrm{P}<0.05, \mathrm{WEV}+\mathrm{NP}$-treated vs. WEV-treated.

Fig. 5. The effects of WEV and WEV+NP on the levels of free radicals in breast cancer cells. The levels of ROS, hydroperoxide and nitric oxide were measured in the lysates of breast cancer cells after treatment with vehicle, NP, WEV or WEV+NP. The accumulated data for 80 patients are expressed as the mean levels of free radical \pm SEM for vehicle-treated cancer cells (closed black bars), NP-treated cells (hatched bars), WEV-treated cells (closed gray bars) and WEV+NP-treated cells (dotted bars). P < 0.05, WEV-treated vs. control; \#P $<0.05$, WEV+NP-treated vs. control; $+\mathrm{P}<0.05$, WEV+NP-treated vs. WEV-treated.

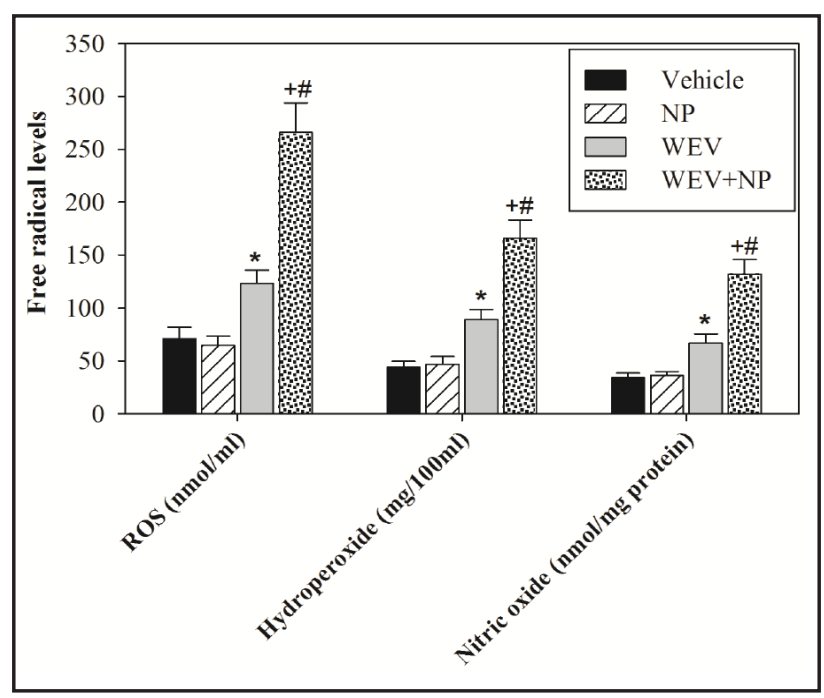

Treatment with WEV and WEV+NP induces apoptosis in breast cancer cells via direct activation of caspase activity

Because caspases are active mediators of apoptosis, the activities of caspase-3, caspase-8 and caspase- 9 were monitored in the breast cancer cell lysates after treatment with vehicle, NP, WEV and WEV+NP. The accumulated data for different patients $(n=70)$ indicated that the levels of caspase-3, caspase-8 and caspase- 9 activities were significantly $(P<0.05)$ increased in the breast cancer cells after treatment with WEV and WEV+NP compared with the vehicleand NP-treated cells (Fig. 4). The effect of WEV+NP on the levels of caspase-3, caspase-8 and caspase- 9 activities was greater than that of WEV alone.

Treatment with WEV and WEV+NP induces the generation of ROS, hydroperoxide and nitric oxide

Tumor cells contain fewer radical scavengers than do normal cells, and free radicals have been shown to participate in the mechanism of anticancer therapeutic agents. Therefore, the generation of a large quantity of free radicals in tumor tissues may represent 
a future anticancer therapy. Therefore, we measured the levels of free radicals in breast cancer cell lysates after treatment with vehicle, NP, WEV or WEV+NP. The accumulated data for different patients $(n=70)$ indicated that the breast cancer cells exhibited a significant $(\mathrm{P}<0.05)$ elevation in the levels of ROS, hydroperoxide and nitric oxide after treatment with WEV alone and WEV+NP compared with treatment with vehicle and NP alone (Fig. 5). Although treatment of breast cancer cells with NP alone had no effect on the generation of free radicals, the effect of WEV was strongly enhanced in combination with NP.

\section{Discussion}

In this study, we investigated the impact of snake venom, either alone or in combination with silica nanoparticles, on breast cancer cell growth and survival. We used cells isolated from female patients who were clinically diagnosed with breast cancer. We demonstrated that WEV alone and in combination with silica nanoparticles inhibited the growth of breast cancer cells in a dose- and time-dependent manner. Moreover, the combination of WEV and NP enhanced the effect of WEV on the cancer cells. The $\mathrm{IC}_{50}$ values of WEV and WEV+NP for the inhibition of the growth of breast cancer cells were $50 \mathrm{ng} / \mathrm{ml}$ and $20 \mathrm{ng} / \mathrm{ml}$, respectively; these values are identical to the values we reported previously for the MDA-MB-231 and MCF-7 breast cancer cell lines [15]. Furthermore, at their IC50s, WEV and WEV+NP caused growth inhibition of breast cancer cells without affecting the viability of non-tumorigenic normal breast epithelial cells (MCF-10) or human normal breast cancer tissue. In addition, we and other researchers have reported the antitumor effects of snake venoms and their ability to induce apoptosis in many cancer cells [16-23].

Furthermore, we recently demonstrated the therapeutic efficacy and molecular mechanisms of WEV+NP in the treatment of breast cancer- and prostate cancer-bearing experimental mouse models [24]. Therefore, in the present study we further investigated the effects of WEV+NP on the human breast cancer cells.

Uncontrolled proliferation is significant in cell turnover and tumorigenesis. Therefore, we monitored the effects of WEV alone or in combination with NP on the proliferation of breast cancer cells with a CFSE dilution assay followed by flow cytometry analysis. Our data revealed that WEV alone and in combination with NP inhibited the proliferation of breast cancer cells. Uncontrolled proliferation is significant in cell turnover and tumorigenesis; thus agents that are able to inhibit proliferation may be useful as chemotherapeutic agents against breast cancer. Our data are consistent with Dowsett et al., who reported that increased apoptosis and decreased proliferation are common factors in the biological response of breast cancer to chemotherapy and endocrine therapy [25]. Positive clinical responses are associated with reduced proliferation during chemotherapy, endocrine therapy and chemoendocrine therapy. Anticancer agents may alter the regulation of the cell cycle machinery, resulting in cellular arrest at different phases of the cell cycle and thereby reducing the growth and proliferation of cancerous cells; cell cycle arrest may even induce apoptosis [26]. Several drugs have been designed to synthetically activate caspases, including peptides that contain the arginine-glycine-aspartate motif. These peptides are pro-apoptotic and have the ability to directly induce auto-activation of procaspase 3. They have also been shown to lower the activation threshold of caspase enzymes or activate caspases, contributing to an increase in the drug sensitivity of cancer cells [27]. Our data reveal that WEV alone and in combination with NP increased the activities of caspase-3, caspase-8 and caspase- 9 in breast cancer cells. Tandon et al concluded that oxidative stress may have potential therapeutic applications in the development of anticancer drugs [28]. Potential anticancer drugs acting by this novel mechanism may prove to be useful in the future. Therefore, we measured the levels of ROS, NO and hydroperoxide, revealing an increase in the levels of free radicals after treatment with WEV and WEV+NP. Generally, chemotherapeutic drugs attack both normal and tumor cells non-specifically and may causing life-threatening side effects, necessitating the 
targeted delivery of drugs to tumors [29]. Indeed, the therapeutic molecule must generally cross one or more biological membranes before diffusing through the plasma membrane to finally gain access to the appropriate organelle where the biological target is located. For a drug whose target is located intracellularly, deviating from this ideal path may not only decrease the drug's efficiency but also entail side effects and toxicity. For these reasons, the design of carriers small enough to ferry the active substance to the target cell and the relevant subcellular compartment was proposed more than 30 years ago [30]. Various types of nanoparticles, such as liposomes, polymeric micelles, dendrimers, superparamagnetic iron oxide crystals and colloidal gold, have been employed in targeted therapies for cancer $[31,32]$. Nanocarriers offer unique possibilities to overcome cellular barriers to improve the delivery of various drug candidates [26]. Recent studies have demonstrated efficient tumor targeting by nanoparticles through an enhanced permeability and retention effect [33-35]. The delivery of drug-loaded nanoparticles has achieved success in the treatment of advanced thyroid cancer and breast carcinoma [36, 37]. Here, while venom-free nanoparticles had no effect on breast cancer cells, the combination of WEV with nanoparticles increased the ability of WEV to kill breast cancer cells by 2-fold compared with WEV alone. Despite great interest in using nanoparticles in biomedical applications, a clear understanding of their cellular uptake and transport is still lacking. Nanoparticles appear to translocate across cells via clathrin- and macropinocytosis-mediated endocytosis [38]. The nanoparticles were also shown to be stable within the cytoplasm for at least $24 \mathrm{~h}$ and did not colocalize within the endosomal pathway. Furthermore, nanoparticle uptake was inhibited approximately $50 \%$ by genistein, an inhibitor of the caveolae-mediated pathway [39]. However, the clathrinmediated endocytosis and macropinocytosis pathways were reduced by 17 and $24 \%$, respectively, in the presence of the respective inhibitors. These findings suggest that PLL-gPEG-DNA nanoparticles enter by several pathways and might therefore be an efficient and versatile tool to deliver therapeutic DNA [40]. Therefore, the combination of nanoparticles with WEV significantly enhanced the antitumor effects of WEV. Snake venom is a complex mixture of many substances, including toxins, enzymes, growth factors, activators and inhibitors, with a wide spectrum of biological activities. Furthermore, synergistic actions between the components of WEV likely contribute to the antitumor effects and the mechanisms that we observed. Subsequently, fractionation of the WEV components and studies of the combination of each fraction with NP are underway. This study revealed the unique biological effects of WEV and WEV+NP on breast cancer cells, which may permit these compounds to be utilized in treatments for breast cancer.

\section{Disclosure Statement}

The authors declare that they have no conflicts of interest, state that the manuscript has not been published or submitted elsewhere, state that the work complies with Ethical Policies of the Journal and the work has been conducted under internationally accepted ethical standards after relevant ethical review.

\section{Abbreviations}

Nanoparticles (NP); reactive oxygen species (ROS); Walterinnesia aegyptia venom (WEV); Walterinnesia aegyptia venom combined with nanoparticles (WEV+NP).

\section{Acknowledgments}

The authors acknowledge Professor Dr. Mohamed Khalid Al-Sadoon for kindly providing us the snake venom. The authors also acknowledge Dr. Ahmed El-Toni at the King 
Badr et al.: Snake Venom-Loaded Silica Nanoparticles Induce Growth Arrest in Human Breast Cancer Cells

Abdullah Institute for Nanotechnology, King Saud University, for loading venom onto the silica nanoparticles.

\section{References}

- Edlich RF, Winters KL, Lin KY: Breast cancer and ovarian cancer genetics. J Long Term Eff Med Implants 2005;15:533-545.

2 Lorusso G, Rüegg C: New insights into the mechanisms of organ-specific breast cancer metastasis. Semin Cancer Biol 2012;22:226-233.

3 Arai M, Taki K, Iwase H, Takizawa K, Nishimura S: Present status and tasks for genetic testing and risk-reducing surgery in patients with hereditary breast and ovarian cancer. Gan To Kagaku Ryoho 2012;39:525-531.

-4 Markland FS, Sheieh K, Zhou Q, Golubkov V, Sherwin RP, Richters V, Sposto R: A: Novel Snake venom disintegrin that inhibits human ovarian cancer dissemination and angiogenesis in an orthotopic nude mouse model. Haemostasis 2001;31:183-191.

5 Brown S, Khan DR: The treatment of breast cancer using liposome technology. J Drug Deliv 2012;2012:212965.

6 Kim YH, Lee BJ, Lee KJ, Cho JH: A case of pituitary metastasis from breast cancer that presented as left visual disturbance. J Korean Neurosurg Soc 2012;51:94-97.

7 Badr G, Al-Sadoon MK, El-Toni AM, Daghestani M: Walterinnesia aegyptia venom combined with silica nanoparticles enhances the functioning of normal lymphocytes through PI3K/AKT, NFKB and ERK signaling. Lipids Health Dis 2012;11:27.

-8 Son DJ, Park MH, Chae SJ, Moon SO, Lee JW, Song HS, Moon DC, Kang SS, Kwon YE, Hong JT: Inhibitory effect of snake venom toxin from Vipera lebetina turanica on hormone-refractory human prostate cancer cell growth: induction of apoptosis through inactivation of nuclear factor kappaB. Mol Cancer Ther 2007;6:675-683.

-9 Barratt G: Colloidal drug carriers: achievements and perspectives. Cell Mol Life Sci 2003;60:21-37.

10 Cereghetti GM, Scorrano L: The many shapes of mitochondrial death. Oncogene 2006;7;25:4717-4724.

11 Debatin KM, Krammer PH: Death receptors in chemotherapy and cancer. Oncogene 2004;12;23:2950-2966.

12 Green D, Kroemer G: The central executioners of apoptosis: caspases or mitochondria? Trends Cell Biol 1998;8:267-271.

13 Haynes CM, Titus EA, Cooper AA: Degradation of misfolded proteins prevents ER-derived oxidative stress and cell death. Mol Cell 2004;15:767-776.

14 Balaban RS, Nemoto S, Finkel T: Mitochondria, oxidants and aging. Cell 2005;120:483-495.

15 Al-Sadoon MK, Abdel-Maksoud MA, Rabah DM, Badr G: Induction of apoptosis and growth arrest in human breast carcinoma cells by a snake (Walterinnesia aegyptia) venom combined with silica nanoparticles: crosstalk between Bcl2 and caspase 3. Cell Physiol Biochem. 2012;30:653-665.

16 Park MH, Jo MR, Won D, Song HS, Han SB, Song MJ, Hong JT: Snake venom toxin from Vipera lebetina turanica induces apoptosis in colon cancer cells via upregulation of ROS- and JNK-mediated death receptor expression. BMC Cancer 2012;12:228.

17 Song JK, Jo MR, Park MH, Song HS, An BJ, Song MJ, Han SB, Hong JT: Cell growth inhibition and induction of apoptosis by snake venom toxin in ovarian cancer cell via inactivation of nuclear factor $\mathrm{\kappa B}$ and signal transducer and activator of transcription 3. Arch Pharm Res 2012;35:867-876.

-18 Al-Sadoon MK, Rabah DM, Badr G: Enhanced anticancer efficacy of snake venom combined with silica nanoparticles in a murine model of human multiple myeloma: molecular targets for cell cycle arrest and apoptosis induction. Cell Immunol 2013;284:129-138.

19 Sayed D, Al-Sadoon MK, Badr G: Silica nanoparticles sensitize human multiple myeloma cells to snake (Walterinnesia aegyptia) venom-induced apoptosis and growth arrest. Oxid Med Cell Longev 2012;2012:386286.

20 Badr G, Al-Sadoon MK, Abdel-Maksoud MA, Rabah DM, El-Toni AM: Cellular and molecular mechanisms underlie the anti-tumor activities exerted by Walterinnesia aegyptia venom combined with silica nanoparticles against multiple myeloma cancer cell types. PLoS One 2012;7:e51661. 
Badr et al.: Snake Venom-Loaded Silica Nanoparticles Induce Growth Arrest in Human Breast Cancer Cells

21 Badr G, Al-Sadoon MK, Rabah DM, Sayed D: Snake (Walterinnesia aegyptia) venom-loaded silica nanoparticles induce apoptosis and growth arrest in human prostate cancer cells. Apoptosis 2013;18:300314.

22 Kollipara PS, Won do H, Hwang CJ, Jung YY, Yoon HS, Park MH, Song MJ, Song HS, Hong JT: Enhanced AntiCancer Effect of Snake Venom Activated NK Cells on Lung Cancer Cells by Inactivation of NF- $\mathrm{KB}$. Biomol Ther (Seoul) 2014;22:106-113.

23 Calderon LA, Sobrinho JC, Zaqueo KD, de Moura AA, Grabner AN, Mazzi MV, Marcussi S, Nomizo A, Fernandes CF, Zuliani JP, Carvalho BM, da Silva SL, Stábeli RG, Soares AM: Antitumoral activity of snake venom proteins: new trends in cancer therapy. Biomed Res Int 2014;2014:203639.

24 Badr G, Al-Sadoon MK, Rabah DM: Therapeutic efficacy and molecular mechanisms of snake (Walterinnesia aegyptia) venom-loaded silica nanoparticles in the treatment of breast cancer- and prostate cancer-bearing experimental mouse models. Free Radic Biol Med 2013;65:175-189.

25 Bruckheimer EM, Kyprianou N: Apoptosis in prostate carcinogenesis. A growth regulator and a therapeutic target. Cell Tissue Res 2000;301:153-162.

26 Dowsett M, Archer C, Assersohn L, Gregory RK, Ellis PA, Salter J, Chang J, Mainwaring P, Boeddinghaus I, Johnston SRD, Powles TJ, Smith IE: Clinical studies of apoptosis and proliferation in breast cancer. Endocrine-Related Cancer 1999;6:25-28.

27 Hu H, Ahn NS, Yang X, Lee YS, Kang KS: Ganoderma lucidum extract induces cell cycle arrest and apoptosis in MCF-7 human breast cancer cell. Int J Cancer 2002;102:250-253.

28 Philchenkov A, Zavelevich M, Kroczak TJ, Los M: Caspases and cancer: mechanisms of inactivation and new treatment modalities. Exp Oncol 2004;26:82-97.

29 Tandon VR, Sharma S, Mahajan A, Bardi GH. Oxidative Stress: A Novel Strategy in Cancer Treatment. JK Science 2005; 7:57-59.

-30 Kolluru LP, Rizvi SA, D'Souza M, D'Souza MJ. Formulation development of albumin based theragnostic nanoparticles as a potential delivery system for tumor targeting. J Drug Target 2013;21:77-86.

31 Hillaireau H, Couvreur P: Nanocarriers' entry into the cell: relevance to drug delivery. Cell Mol Life Sci 2009;66:2873-2896.

32 Wang J, Sui M, Fan W: Nanoparticles for tumor targeted therapies and their pharmacokinetics. Curr Drug Metab 2010;11:129-141.

-33 Haque F, Shu D, Shu Y, Shlyakhtenko LS, Rychahou PG, Evers BM, Guo P. Ultrastable synergistic tetravalent RNA nanoparticles for targeting to cancers. Nano Today 2012;7:245-257.

34 Nam HY, Kwon SM, Chung H, Lee SY, Kwon SH, Jeon H, Kim Y, Park JH, Kim J, Her S, Oh YK, Kwon IC, Kim K, jeong SY: Cellular uptake mechanism and intracellular fate of hydrophobically modified glycol chitosan nanoparticles. J Control Release 2009;135:259-267.

35 Koo H, Huh MS, Sun IC, Yuk SH, Choi K, Kim K, Kwon IC: In vivo targeted delivery of nanoparticles for theranosis. Acc Chem Res 2011;44:1018-1028.

-36 Lim SM, Kim TH, Jiang HH, Park CW, Lee S, Chen X, Lee KC: Improved biological half-life and anti-tumor activity of TNF-related apoptosis-inducing ligand (TRAIL) using PEG-exposed nanoparticles. Biomaterials 2011;32:3538-3546.

- 37 Koppolu B, Bhavsar Z, Wadajkar AS, Nattama S, Rahimi M, Nwariaku F, Nguyen KT: Temperature-sensitive polymer-coated magnetic nanoparticles as a potential drug delivery system for targeted therapy of thyroid cancer. J Biomed Nanotechnol 2012;8:983-990.

- 38 Zeng X, Zhang Y, Nyström AM: Endocytic uptake and intracellular trafficking of bis-MPA based hyperbranched copolymer micelles in breast cancer cells. Biomacromolecules 2012;13:2814-3822.

39 Chen D, Tang Q Li X, Zhou X, Zang J, Xue WQ, Xiang JY, Guo CQ: Biocompatibility of magnetic Fe(3)O(4) nanoparticles and their cytotoxic effect on MCF-7 cells. Int J Nanomed 2012;7:4973-4982.

-40 Lühmann T, Rimann M, Bittermann AG, Hall H: Cellular uptake and intracellular pathways of PLL-g-PEGDNA nanoparticles. Bioconjug Chem 2008;19:1907-1916. 